\title{
Spectrum-Effect Relationship between HPLC Fingerprints and Antioxidant Activity of Yangyin Tongnao Prescription
}

\author{
Li Yu ${ }^{\mathbb{D}},{ }^{1}$ Yangyang Zhang $\mathbb{D}^{1},{ }^{1}$ Xixi Zhao, ${ }^{2}$ Yu He, ${ }^{3}$ Haofang Wan, ${ }^{4}$ Haitong Wan $\mathbb{D}^{1}$, \\ and Jiehong Yang $\mathbb{D}^{2}$ \\ ${ }^{1}$ School of Life Sciences, Zhejiang Chinese Medical University, Hangzhou, Zhejiang 310053, China \\ ${ }^{2}$ School of Basic Medical Sciences, Zhejiang Chinese Medical University, Hangzhou, Zhejiang 310053, China \\ ${ }^{3}$ School of Pharmaceutical Sciences, Zhejiang Chinese Medical University, Hangzhou, Zhejiang 310053, China \\ ${ }^{4}$ Academy of Chinese Medical Sciences, Zhejiang Chinese Medical University, Hangzhou, Zhejiang 310053, China \\ Correspondence should be addressed to Haitong Wan; whtong@126.com and Jiehong Yang; yjhong@zcmu.edu.cn
}

Received 10 October 2020; Revised 29 March 2021; Accepted 19 May 2021; Published 21 June 2021

Academic Editor: Eduardo Dellacassa

Copyright (c) $2021 \mathrm{Li} \mathrm{Yu}$ et al. This is an open access article distributed under the Creative Commons Attribution License, which permits unrestricted use, distribution, and reproduction in any medium, provided the original work is properly cited.

\begin{abstract}
Yangyin Tongnao (YYTN) prescription is used as a traditional Chinese herbal formula, and it has antioxidant activity that mainly contributes in the treatment of cardiovascular and cerebrovascular diseases. However, the compounds related to its antioxidant activity are still unknown. In the present study, the fingerprints of YYTN extracts under different extraction conditions were obtained by high performance liquid chromatography (HPLC) to identify the common peaks to all the samples processed. A 2,2diphenyl-1-picrylhydrazyl (DPPH) radical scavenging activity assay and ferric reducing antioxidant power (FRAP) assay were carried out to evaluate the antioxidant activity of the extracts. Spectrum-effect relationship between HPLC fingerprints and antioxidant activity of YYTN was assessed by Pearson product-moment correlation coefficient (PPMCC) and multiple linear regression analysis (MLRA). The results showed that peaks 5, 6, 13, 15, and 24 of the fingerprints were closely connected to antioxidant activity. Five peaks were identified: vanillic acid (P5), puerarin (P7), ferulic acid (P13), daidzein (P21), and formononetin (P23). Our study successfully established the spectrum-effect relationship between HPLC fingerprints and antioxidant activity of YYTN, which provided a general method for establishing quality standards with a combination of chromatography and antioxidant activity.
\end{abstract}

\section{Introduction}

Yangyin Tongnao prescription is a traditional Chinese herbal formula composed of six kinds of Chinese herbal medicines, Radix Rehmanniae, Radix Astragali, Puerariae Lobatae Radix, Ligustici Chuanxiong Rhizoma, Dendrobii Caulis, and Hirudo. However, four kinds of these Chinese herbal medicines except Dendrobii Caulis and Hirudo have been studied as a simplified formula in a lot of research $[1,2]$. The proportions of herbal composition of Yangying Tongnao (YYTN) simplified prescription are shown in Table 1 [3]. The efficacy of YYTN has been evaluated by clinical trials and animal experiments. According to the Chinese medicine, it has the effects of nourishing yin and replenishing qi (deficiency of yin or qi is a kind of syndrome; deficiency of yin usually appears hot and bothered, and deficiency of qi has a feeling of weariness). In the Western medicine, it could promote blood circulation, especially in the treatment of cardiovascular and cerebrovascular diseases [4]. Previous researches have proved that YYTN had the functions of inhibiting neuronal apoptosis, improving haematological disorders [5], and is related with antioxidant and anti-inflammatory properties [6]. As a traditional Chinese herbal formula, YYTN can also improve the neurological deficits after focal cerebral ischemic infarction in rats [3]. At the same time, it was found that YYTN has protective effect on oxidative stress-induced cerebral ischemia injury in vivo through animal experiments [7]. However, there does not 
TABLE 1: The herbal composition of YYTN simplified prescription.

\begin{tabular}{lcccc}
\hline Pharmaceutical name & Botanical name & Botanical family & Quantity (g) & Employed plant part \\
\hline Radix Rehmanniae & Rehmannia glutinosa Libosch. & Scrophulariaceae & 1 & Tuberous root \\
Radix Astragali & Astragalus membranaceus (Fisch.) Bge. & Leguminosae & 1 & Root \\
Puerariae Lobatae Radix & Pueraria lobata (Willd.) Ohwi & Lamiaceae & 1.2 & Root \\
Ligustici Chuanxiong Rhizoma & Ligusticum chuanxiong Hort. & Apiaceae & 0.67 & Root and rhizome \\
\hline
\end{tabular}

seem to be any report about the relationship between active ingredients and pharmaceutical efficacy of YYTN.

Antioxidants play a crucial role in preventing various serious diseases caused by oxidative stress damage. At present, several methods have been applied to evaluate the antioxidant activity, including assays for ferric reducing antioxidant power (FRAP) [8-10], 2, 2-diphenyl-1-picrylhydrazyl (DPPH) radical scavenging [11-13], and hydroxyl radical scavenging $[14,15]$. Therefore, a growing number of researchers are paying attention to the search for natural compounds from plants or vegetal drugs with antioxidant properties [16-18]. However, the compounds of YYTN responsible for its antioxidant activity are unclear.

Nowadays, spectrum-effect is used to study the relationship between components and efficacy of traditional Chinese medicines. For example, the main vasorelaxant components of rosemary have been found by using the chemical spectrumeffect relationship [19]. HPLC fingerprint and bioactivity verification were used to investigate the potential correlation between the chemical components and pharmaceutical effects $[20,21]$. It is a representative method to find the main active components in complex traditional Chinese herbal formulas based on the study of spectrum-activity relationship.

There are three major categories of data processing methods for spectrum-effect relationships, such as correlation analysis, cluster analysis, and regression analysis. Correlation analysis is a statistical method for studying the degree of closeness between variables [22], including grey relational analysis (GRA) and Pearson product-moment correlation coefficient (PPMCC). Cluster analysis is one of the important techniques in data mining and exploratory data analysis [23], including hierarchical clustering analysis (HCA) and nonhierarchical clustering analysis (N-HCA). Regression analysis refers to a statistical analysis method to determine the quantitative relationship between two or more variables [24], including multiple linear regression analysis (MLRA) and partial least squares (PLS). Therefore, GRA, PPMCC, PLS, and MLRA are applied to study the spectrum-effect relationship in the correlation analysis between the components and the efficacy of Chinese formulas [25-29].

In our study, HPLC fingerprints were combined with the data of the 2,2-diphenyl-1-picrylhydrazyl (DPPH) radical scavenging and ferric reducing antioxidant power (FRAP) assays to study the spectrum-effect relationship of YYTN using PPMC and MLRA. It lays the foundation for the pharmacokinetics substantial basis of YYTN.

\section{Materials and Methods}

2.1. Materials and Reagents. Radix Rehmanniae, Radix Astragali, Puerariae Lobatae Radix, and Chuanxiong
Rhizoma were purchased from Traditional Chinese Medicine Pieces Co., Ltd. of Zhejiang Chinese Medical University (Hangzhou, China) and identified by Professor Shengwu Huang, Zhejiang Chinese Medical University (Hangzhou, China). HPLC-grade methanol and acetonitrile were obtained from Tedia Company, Inc. (Fairfield, OH, USA). Water was purified with a Millipore purification system (Millipore Co., Ltd, Billerica, MA, USA). DPPH was purchased from Shanghai Biochemical Co., Ltd. (Shanghai, China). FRAP kit was purchased from Nanjing Institute of Bioengineering (Nanjing, China). The reference standards of vanillic acid $(\geq 99.0 \%)$, puerarin $(\geq 98.0 \%)$, ferulic acid $(\geq 98.0 \%)$, daidzein $(\geq 99.0 \%)$, and formononetin $(\geq 98.0 \%)$ were purchased from Shanghai Yuanye Co., Ltd. (Shanghai, China).

\subsection{HPLC Fingerprints}

2.2.1. Chromatographic Conditions. HPLC analysis was performed using a Waters 2695 HPLC equipped with 2498 UV detector. The chromatographic separation was performed on a Hypersil BDS C18 column $(4.6 \mathrm{~mm} \times 300 \mathrm{~mm}$, $5 \mu \mathrm{m})$ maintained at $25^{\circ} \mathrm{C}$. The mobile phase consisted of acetonitrile $(A)$ and $0.5 \%$ formic acid water $(B)$ with the following optimized gradient elution: $0 \mathrm{~min}$ : $2 \% \mathrm{~A}$; $10 \mathrm{~min}$ : $10 \%$ A; $29 \mathrm{~min}: 12 \%$ A; $35 \mathrm{~min}: 15 \%$ A; $50 \mathrm{~min}: 25 \%$ A; $65 \mathrm{~min}$ : $60 \% \mathrm{~A} ; 70 \mathrm{~min}: 90 \% \mathrm{~A}$. The flow rate was $1.0 \mathrm{~mL} / \mathrm{min}$ with an injection volume of $10 \mu \mathrm{L}$, and the detection wavelength was set at $280 \mathrm{~nm}$.

2.2.2. Preparation of Standard Solutions. The mixed reference standard solution was prepared by adding $3 \mathrm{mg}$ of vanillic acid, $36 \mathrm{mg}$ of puerarin, $2.4 \mathrm{mg}$ of ferulic acid, $6 \mathrm{mg}$ of daidzein, and $1.8 \mathrm{mg}$ of formononetin in a $10 \mathrm{~mL}$ volumetric flask and dissolved in 50\% methanol to obtain final concentrations of $0.3,3.6,0.24,0.6$, and $0.18 \mathrm{mg} / \mathrm{mL}$, respectively. Then the solution was filtered through a $0.22 \mu \mathrm{m}$ microporous membrane, and 50\% methanol was added to final concentrations of $0.15,0.075,0.05,0.0375$, and $0.03 \mathrm{mg} /$ $\mathrm{mL}$ of vanillic acid; $1.8,0.9,0.6,0.45$, and $0.36 \mathrm{mg} / \mathrm{mL}$ of puerarin; $0.12,0.06,0.04,0.03$, and $0.024 \mathrm{mg} / \mathrm{mL}$ of ferulic acid; $0.3,0.15,0.1,0.075$, and $0.06 \mathrm{mg} / \mathrm{mL}$ of daidzein; 0.09 , $0.045,0.03,0.0225$, and $0.018 \mathrm{mg} / \mathrm{mL}$ of formononetin for HPLC analysis.

2.2.3. Preparation of Sample Solutions. The four herbs were crushed into crude grains. Accurately weighed $1.00 \mathrm{~g}$ of Radix Rehmanniae, $1.00 \mathrm{~g}$ of Radix Astragali, $1.20 \mathrm{~g}$ of Puerariae Lobatae Radix, and 0.67 g of Chuanxiong Rhizoma crude grains were mixed, and aqueous extracts were 
prepared using ultrasound-assisted extraction according to Tables 2 and 3 . All the solutions were filtered through a $0.22 \mu \mathrm{m}$ microporous membrane and stored at $4^{\circ} \mathrm{C}$ until analysis.

2.2.4. Validation of Methodology. Method precision was evaluated by five replicate injections of one sample, while repeatability was evaluated by five samples prepared independently from the same initial conditions. For the stability study, sample solutions were stored at room temperature and analyzed at $0,2,4,8,12$, and $24 \mathrm{~h}$, respectively.

\subsubsection{Peak Identification and Evaluation of Fingerprints.} The reference standard solutions were injected into the HPLC system, and the chromatographic peaks in the sample solutions were identified according to the retention time of the reference substances.

Each sample solution and each standard solution was analyzed with the HPLC optimized condition to obtain the HPLC fingerprints. The fingerprints were matched automatically by the Similarity Evaluation System for Chromatographic Fingerprint of Traditional Chinese Medicine [30] (Version 2012A). Then the reference fingerprint was formed by the system using the median method from the comparison of nine samples, and the similarity values between the reference and nine samples were calculated using this software.

\subsection{Antioxidant Activity Determination}

2.3.1. DPPH Assay. DPPH radical scavenging activity was determined according to Zhou et al. [31] with slight modifications. Briefly, $100 \mu \mathrm{L}$ of $0.4 \mathrm{mM}$ DPPH anhydrous ethanol solution and $100 \mu \mathrm{L}$ of each sample solution at various concentrations were added to the 96 microplate. After incubation for $30 \mathrm{~min}$ at room temperature in the dark, the absorbance of the mixture was measured at $517 \mathrm{~nm}$ (SpectraMax Plus384 Absorbance Microplate Reader, Molecular Devices, USA). DPPH radical scavenging activity was calculated according to the following equation:

$$
\text { scavenging percentage }(\%)=\left(1-\frac{A_{i}-A_{j}}{A_{0}}\right) \times 100 \text {, }
$$

where $A_{\mathrm{i}}$ was the absorbance of sample $+\mathrm{DPPH} ; A_{\mathrm{j}}$ was the absorbance of sample + anhydrous ethanol; $A_{0}$ was the absorbance of anhydrous ethanol + DPPH.

2.3.2. FRAP Assay. The total antioxidant activity was evaluated by FRAP assay [32]. The FRAP solution was prepared by mixing $1.50 \mathrm{~mL}$ of $300 \mathrm{mmol} / \mathrm{L}$ acetate buffer $(\mathrm{pH}=3.6)$ with $0.15 \mathrm{~mL}$ of $10 \mathrm{mmol} / \mathrm{L}$ TPTZ solution in $40 \mathrm{mmol} / \mathrm{L} \mathrm{HCl}$ and $0.15 \mathrm{~mL}$ of $20 \mathrm{mmol} / \mathrm{L} \mathrm{FeCl}_{3}$ solution, incubated for $30 \mathrm{~min}$ at $37^{\circ} \mathrm{C}$ in the dark. A fresh $100 \mathrm{mM}$ $\mathrm{FeSO}_{4}$ solution was prepared from $\mathrm{FeSO}_{4} \cdot 7 \mathrm{H}_{2} \mathrm{O}$ standard material. Then $5 \mu \mathrm{L}$ of the sample and $180 \mu \mathrm{L}$ of FRAP solution were added to the 96 microplate and allowed to react in the dark for $6 \mathrm{~min}$ at room temperature, and the
TABLE 2: Independent variables and their levels.

\begin{tabular}{lccc}
\hline Factors & \multicolumn{3}{c}{ Levels } \\
& 1 & 2 & 3 \\
\hline Temperature $\left({ }^{\circ} \mathrm{C}\right)$ & 50 & 60 & 70 \\
Extraction time $(\mathrm{min})$ & 40 & 50 & 60 \\
Solvent-to-material ratio $(\mathrm{mL} / \mathrm{g})$ & 6 & 7 & 8 \\
Deviation & 1 & 2 & 3 \\
\hline
\end{tabular}

absorbance was measured at $593 \mathrm{~nm}$ (SpectraMax Plus384 Absorbance Microplate Reader, Molecular Devices, USA). Aqueous standard solutions of $0.15,0.3,0.6,0.9,1.2$, and $1.5 \mathrm{mmol} / \mathrm{L}$ of $\mathrm{FeSO}_{4}$ were used to generate a calibration curve. The final results were expressed as the equivalent concentration of the $\mathrm{FeSO}_{4}$ standard solution.

\subsection{Spectrum-Effect Relationship}

2.4.1. Pearson Product-Moment Correlation Coefficient. The PPMCC is a statistical method to study the degree of closeness and direction of change between the two variables [33]. In this study, PPMCC was used to calculate the relationship between the peak area values of twenty-four common peaks to all the samples (P1 to P24) in HPLC fingerprints and the antioxidant activities.

2.4.2. Multiple Linear Regression Analysis. To eliminate the problem of multicollinearity that may exist among multiple independent variables, this study used the stepwise regression module in multiple linear regression in SPSS statistical software [34] to analyze the peaks of HPLC fingerprints, $\mathrm{IC}_{50}$ values of DPPH radical scavenging percentage, and the ferric reducing antioxidant power, respectively. Therefore, characteristic peaks with significant correlation among multiple independent variables were selected.

2.5. Statistical Analysis. The $\mathrm{IC}_{50}$ was calculated using the following URL: https://www.aatbio.com/tools/ic50calculator. MLRA and PPMCC were performed by SPSS 25.0 software.

\section{Results}

\subsection{Results of HPLC Fingerprints}

3.1.1. Method Validation. Method validation for HPLC fingerprint results showed that precision of the same sample solution was within the range of $0.19-1.37 \%$ for relative retention time and $0.75-2.67 \%$ for the average peak area of the common peaks to all the samples. Repeatability of the experiment appeared within the range of $0.15-1.04 \%$ for the relative retention time and $0.57-2.48 \%$ for relative peak area of common peaks. Sample stability was $0.11-1.32 \%$ for relative retention time and $0.69-2.74 \%$ for relative peak area of the common peaks to all the samples. All results indicated that the method of HPLC analysis was valid and satisfactory. 
TABLE 3: The orthogonal table of sample solutions.

\begin{tabular}{lcccc}
\hline Run & Temperature $\left({ }^{\circ} \mathrm{C}\right)$ & Extraction time $(\mathrm{min})$ & Solvent-to-material ratio $(\mathrm{mL} / \mathrm{g})$ & Deviation \\
\hline S1 & 1 & 1 & 2 & 1 \\
S2 & 1 & 2 & 3 & 2 \\
S3 & 1 & 3 & 2 & 3 \\
S4 & 2 & 1 & 3 & 3 \\
S5 & 2 & 2 & 1 & 1 \\
S6 & 2 & 3 & 3 & 2 \\
S7 & 3 & 1 & 1 & 3 \\
S8 & 3 & 2 & 2 & 1 \\
S9 & 3 & 3 & & 2 \\
\hline
\end{tabular}

3.1.2. HPLC Fingerprints. The HPLC fingerprints of the extracts of YYTN and their reference fingerprints are shown in Figure 1, and sample and mixed reference solution are shown in Figures 2 and 3, respectively. Twenty-four peaks with good separation and large peak areas were selected as common peaks to all the samples. The average retention time and peak areas of samples and the coefficient of variation (CV) of twenty-four peak areas are listed in Table 4. Values of CV were all more than $8.96 \%$, which indicated that the content of each component of the samples under different extraction conditions varies greatly, especially the unknown compound represented by peak 2 .

3.1.3. Quantitative Analysis of YYTN. After comparing with the reference standard, five of the twenty-four common peaks were identified, which were vanillic acid (P5), puerarin $(P 7)$, ferulic acid (P13), daidzein (P21), and formononetin (P23). As shown in Table 5, quality control was performed on the contents of five compounds in nine samples.

3.1.4. Similarity Analysis of Fingerprints. Each sample fingerprint of YYTN was analyzed by comparing with the reference fingerprint to evaluate the similarity among these samples. Results of similarity values between the reference and the nine samples are shown in Table 6.

\subsection{Results of the Antioxidant Activity Test}

3.2.1. DPPH Assay. The $\mathrm{IC}_{50}$ values of $\mathrm{DPPH}$ radical scavenging activity test are listed in Table 7 . The results revealed that nine extracts of YYTN solution exhibited the $\mathrm{IC}_{50}$ values that appeared within the range of $3.023-4.396 \mathrm{mg} / \mathrm{mL}$. As shown in Table 7, S5 exhibited the most DPPH radical scavenging activity.

3.2.2. FRAP Assay. According to the FRAP kit, the absorbance value of the $\mathrm{FeSO}_{4}$ standard was plotted on the abscissa, and the concentration corresponding to each absorbance value was plotted on the ordinate. The regression equation was as follows: $Y=3.4887 X-0.0291, R^{2}=0.9994$. The results revealed that the nine extracts of YYTN solution varied from 5.331 to $8.245 \mathrm{mmol} / \mathrm{L}$ equivalent to $\mathrm{FeSO}_{4}$. As shown in Table 7, S9 exhibited the highest ferric reducing antioxidant power.

\subsection{Spectrum-Effect Relationship Results}

3.3.1. Pearson Product-Moment Correlation Coefficient. PPMCC analysis is performed with peak areas of twentyfour peaks as independent variables and $\mathrm{IC}_{50}$ values of DPPH radical scavenging percentage and FRAP values as dependent variables. Results of PPMCC are shown in Table 8. The PPMCC represents the correlation between the peak areas and the $\mathrm{IC}_{50}$ values of radical scavenging percentage and the FRAP values. If the PPMCC value was positive, it indicates that there was a positive correlation between independent and dependent variables. Conversely, if the PPMCC value is negative, then there is a negative correlation. The larger the absolute value of PPMCC, the better the correlation.

3.3.2. Multiple Linear Regression Analysis. Using SPSS software, the peak areas of twenty-four common peaks were selected as independent variables and expressed as P1-P24, and $\mathrm{IC}_{50}$ values of DPPH radical scavenging percentage and FRAP values were selected as dependent variables and expressed as $Y 1$ and $Y 2$, respectively. By stepwise regression analysis, the multiple regression equation of $\mathrm{IC}_{50}$ values of $\mathrm{DPPH}$ radical scavenging percentage variable was expressed as $Y 1=0.308-0.004 \times P 5-0.001 \times P 6+0.001 \times P 9-0.002$ $\times P 13-0.004 \times P 15+0.006 \times P 16-0.001 \times P 21+0.011 \times P 24$, and the equation of FRAP was expressed as $Y 2=-1.742+$ $0.011 \times P 5+0.008 \times P 6-\times P 9+0.001 \times P 13-0.026 \times P 15+$ $0.024 \times P 16-0.001 \times P 21+0.044 \times P 24$. These two multivariate linear regression equations represent peaks that were strongly correlated with $\mathrm{IC}_{50}$ values of DPPH radical scavenging percentage and FRAP values. In the regression process, some peaks with no obvious correlation and multicollinearity were eliminated. Finally, the correlation peak was chosen to get the regression equation.

\section{Discussion}

YYTN is composed of Radix Rehmanniae, Radix Astragali, Puerariae Lobatae Radix, and Chuanxiong Rhizoma. Radix Rehmanniae had been found to have anticancer effects through promoting the activation of natural killer cells and dendritic cells $[35,36]$. Radix Astragali has antitumor activity in vitro and in vivo [37]. It also exerts pharmacological activities on the cardiovascular, immune, respiratory, and hepatic systems [38]. Puerariae Lobatae Radix has been 


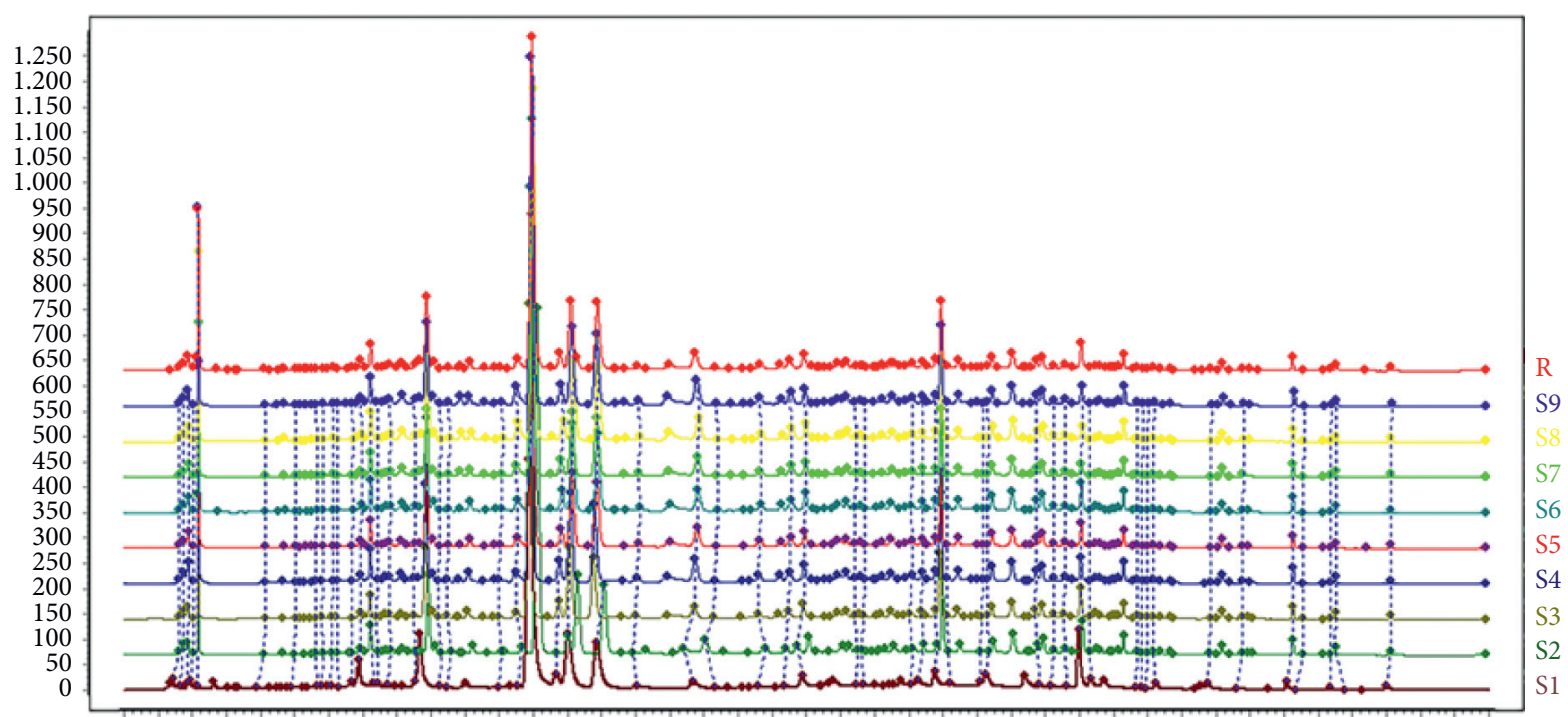

$0 \quad 2 \quad 4 \quad 6 \quad 8101214161820222426283032343638404244464850525456586062646668707274767880$

FIGURE 1: HPLC fingerprints of YYTN and their reference (the detection wavelength was set at $280 \mathrm{~nm}$, and the time is expressed in minutes).

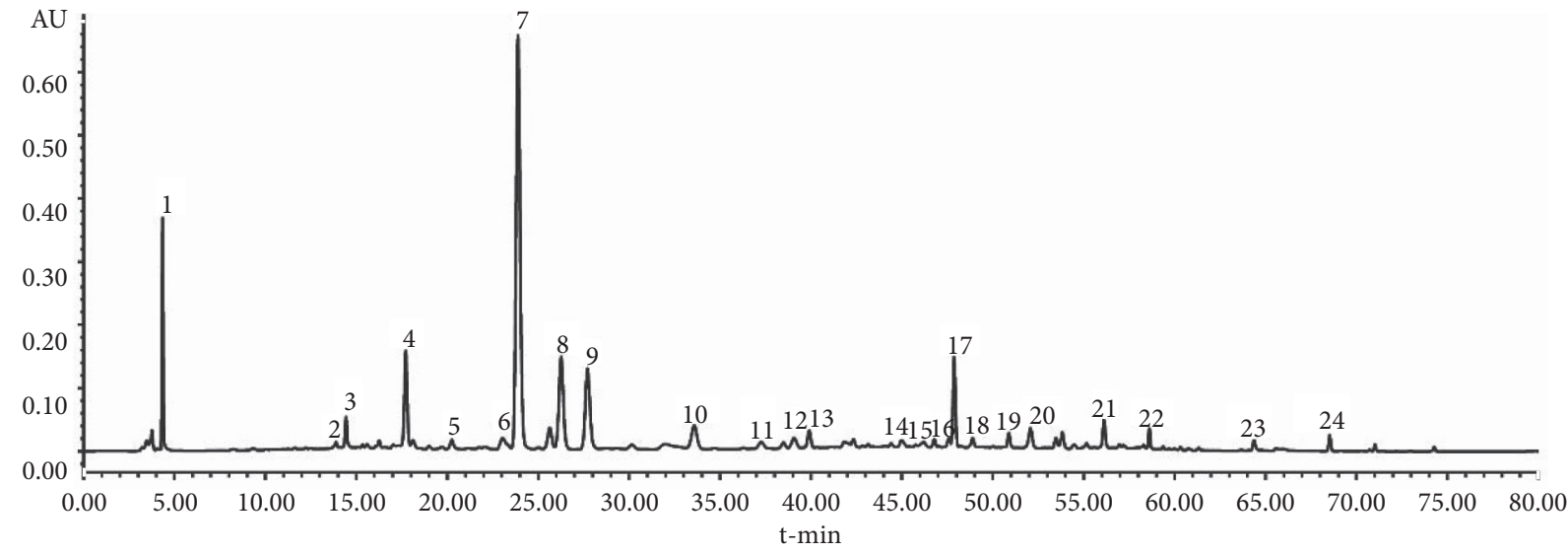

Figure 2: The HPLC fingerprint of YYTN.

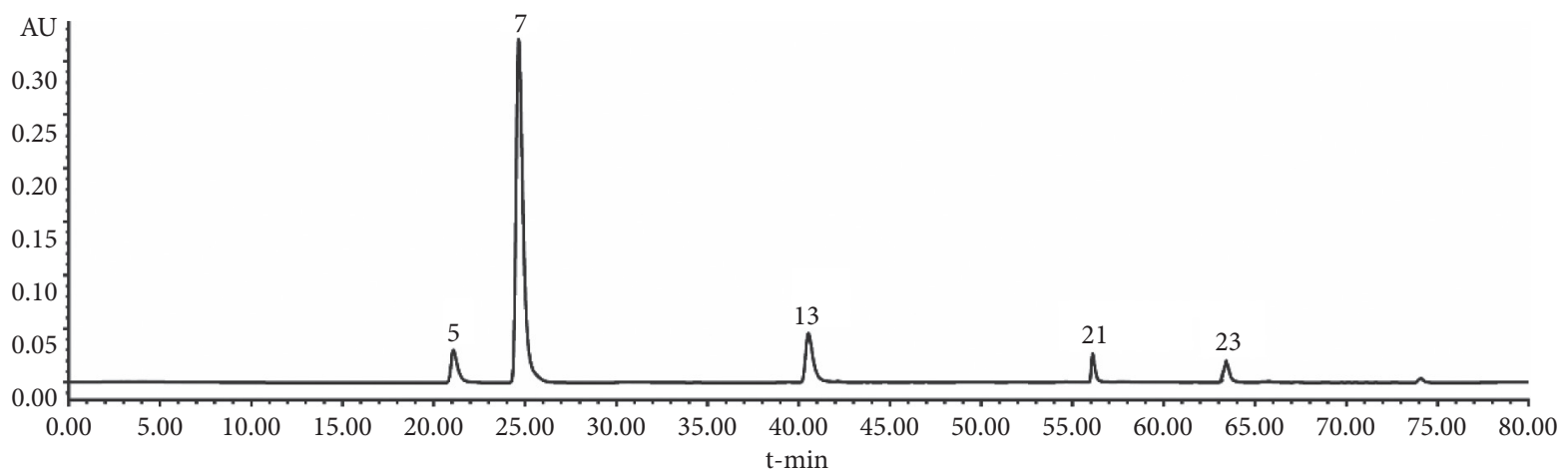

Figure 3: The HPLC fingerprint of mixed reference solution. Vanillic acid (P5), puerarin (P7), ferulic acid (P13), daidzein (P21), and formononetin (P23).

shown to be effective in the treatment of hypertension, cerebral ischemia, and diabetes [39-41]. Chuanxiong Rhizoma has a protective effect on diabetic nephropathy and has anticancer and antioxidant pharmacological activities $[42,43]$. Therefore, YYTN, as the traditional Chinese herbal formula that combines these four Chinese medicines, has 


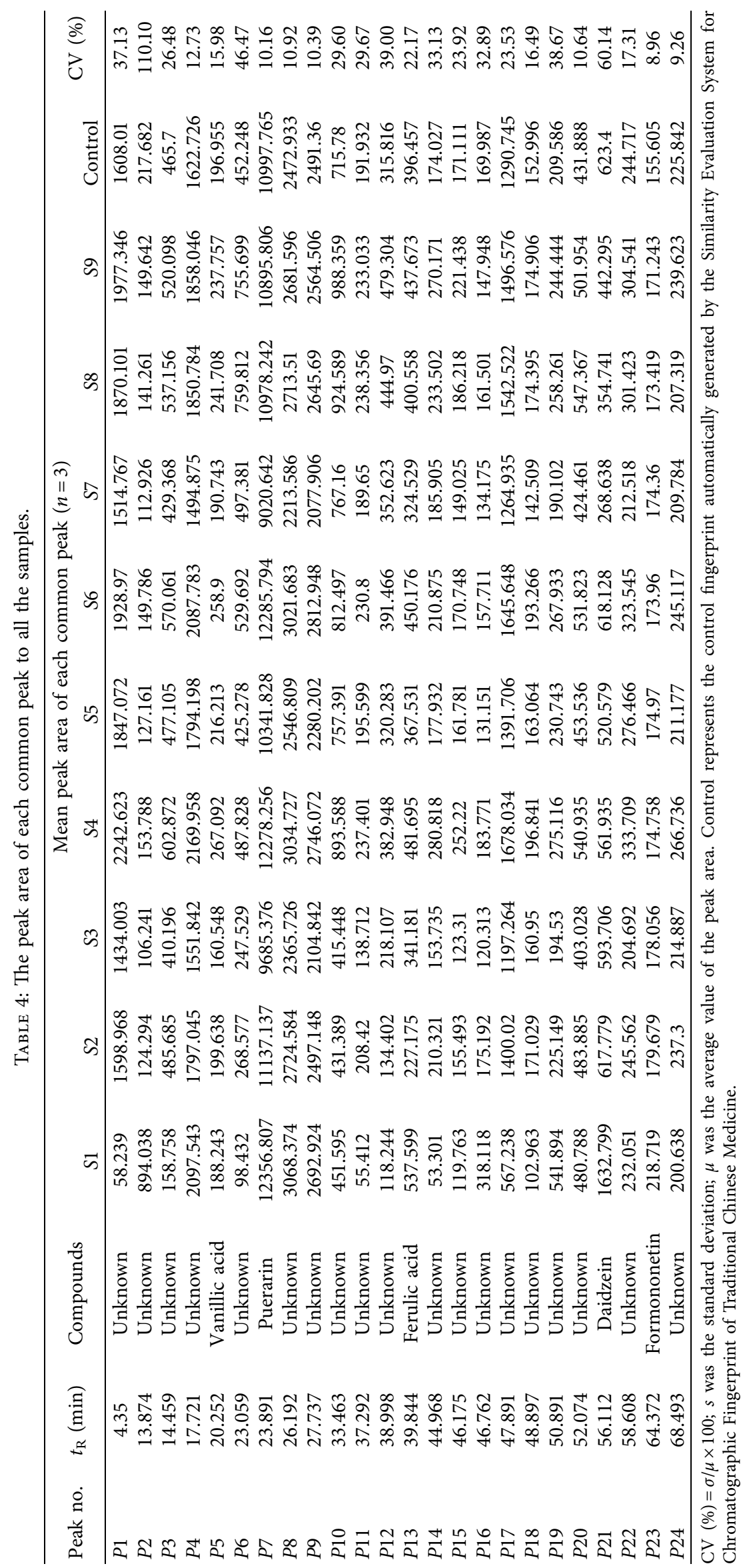


TABLE 5: The content of each group sample.

\begin{tabular}{|c|c|c|c|c|c|c|c|c|c|c|}
\hline \multirow{2}{*}{ Compounds } & \multirow{2}{*}{ Standard curve $\left(R^{2}\right)$} & \multicolumn{9}{|c|}{ The content of each group sample $(\mathrm{mg} / \mathrm{mL})$} \\
\hline & & $S 1$ & $S 2$ & S3 & $S 4$ & S5 & S6 & $S 7$ & S8 & $S 9$ \\
\hline Vanillic acid & $Y=1 \times 10^{7} X-7.89 \times 10^{4}\left(R^{2}=0.999\right)$ & 0.059 & 0.056 & 0.048 & 0.069 & 0.059 & 0.068 & 0.054 & 0.064 & 0.063 \\
\hline Puerarin & $Y=1 \times 10^{7} X-1.30 \times 10^{4}\left(R^{2}=0.999\right)$ & 2.397 & 2.230 & 1.940 & 2.458 & 2.071 & 2.460 & 1.807 & 2.198 & 2.182 \\
\hline Ferulic acid & $Y=3 \times 10^{7} X+6.59 \times 10^{4}\left(R^{2}=0.998\right)$ & 0.024 & 0.021 & 0.018 & 0.028 & 0.013 & 0.026 & 0.017 & 0.022 & 0.025 \\
\hline Daidzein & $Y=8 \times 10^{6} X+9.69 \times 10^{4}\left(R^{2}=0.998\right)$ & 0.124 & 0.130 & 0.124 & 0.116 & 0.106 & 0.130 & 0.043 & 0.064 & 0.086 \\
\hline Formononetin & $Y=2 \times 10^{6} X+1.63 \times 10^{4}\left(R^{2}=0.999\right)$ & 0.155 & 0.163 & 0.162 & 0.159 & 0.159 & 0.158 & 0.158 & 0.157 & 0.155 \\
\hline
\end{tabular}

$R^{2}$ is the square of the correlation coefficient, which can judge the fitting degree of the linear regression line.

TABLE 6: Sample similarity analysis.

\begin{tabular}{lcccccccccc}
\hline & $S 1$ & $S 2$ & $S 3$ & $S 4$ & $S 5$ & $S 6$ & $S 7$ & $S 8$ & $S 9$ & Control \\
\hline$S 1$ & 1 & 0.907 & 0.951 & 0.942 & 0.944 & 0.947 & 0.943 & 0.942 & 0.941 & 0.957 \\
S2 & 0.907 & 1 & 0.956 & 0.954 & 0.954 & 0.955 & 0.953 & 0.952 & 0.952 & 0.964 \\
S3 & 0.951 & 0.956 & 1 & 0.998 & 0.999 & 0.999 & 0.997 & 0.996 & 0.996 & 0.998 \\
S4 & 0.942 & 0.954 & 0.998 & 1 & 1 & 0.999 & 0.999 & 0.998 & 0.998 & 0.998 \\
S5 & 0.944 & 0.954 & 0.999 & 1 & 1 & 1 & 0.999 & 0.999 & 0.999 & 0.998 \\
S6 & 0.947 & 0.955 & 0.999 & 0.999 & 1 & 1 & 0.999 & 0.999 & 0.998 & 0.999 \\
S7 & 0.943 & 0.953 & 0.997 & 0.999 & 0.999 & 0.999 & 1 & 1 & 1 \\
S8 & 0.942 & 0.952 & 0.996 & 0.998 & 0.999 & 0.999 & 1 & 1 & 1 \\
S9 & 0.941 & 0.952 & 0.996 & 0.998 & 0.999 & 0.998 & 1 & 1 & 0.998 \\
Control & 0.957 & 0.964 & 0.998 & 0.998 & 0.998 & 0.999 & 0.998 & 0.997 & 0.997 & 0.997 \\
\hline
\end{tabular}

This analysis was carried out with the software "Similarity Evaluation System for Chromatographic Fingerprint of Traditional Chinese Medicine."

TABLe 7: The results of antioxidant activities $(\bar{x} \pm \mathrm{SD}, n=3)$.

\begin{tabular}{lcc}
\hline Sample no. & $\begin{array}{c}\mathrm{DPPH} \\
\mathrm{IC}_{50}(\mathrm{mg} / \mathrm{mL})\end{array}$ & $\begin{array}{c}\text { FRAP } \\
\mathrm{FeSO}_{4} \text { equivalent }(\mathrm{mmol} / \mathrm{L})\end{array}$ \\
\hline S1 & $3.775 \pm 0.017$ & $6.328 \pm 0.025$ \\
S2 & $4.396 \pm 0.024$ & $6.170 \pm 0.034$ \\
S3 & $3.290 \pm 0.018$ & $5.331 \pm 0.029$ \\
S4 & $3.694 \pm 0.011$ & $7.242 \pm 0.022$ \\
S5 & $3.023 \pm 0.016$ & $6.003 \pm 0.019$ \\
S6 & $3.637 \pm 0.016$ & $7.762 \pm 0.022$ \\
S7 & $3.307 \pm 0.017$ & $7.345 \pm 0.025$ \\
S8 & $3.296 \pm 0.022$ & $7.959 \pm 0.033$ \\
S9 & $3.116 \pm 0.017$ & $8.245 \pm 0.032$ \\
\hline
\end{tabular}

been extensively applied in the treatment of brain injury [3]. However, there are few reports on the HPLC fingerprint and pharmacological efficacy of YYTN.

From Figure 1 and Table 4, there were certain differences in the peak areas between the nine samples under different extraction conditions. However, as shown in Table 6, the similarity value of nine sample fingerprints and reference fingerprints ranged from 0.907 to 0.999 , which indicated that the quality of the YYTN composed of Radix Rehmanniae, Radix Astragali, Puerariae Lobatae Radix, and Chuanxiong Rhizoma was relatively consistent and stable. We identified five compounds in YYTN by comparing Figures 2 and 3: P5 was vanillic acid, $P 7$ was puerarin, $P 13$ was ferulic acid, $P 21$ was daidzein, and $P 23$ was formononetin. Subsequently, we carried out the quantitative determination of these compounds' content. The results are shown in Table 5: the contents of known components in YYTN were puerarin $>$ formononetin $>$ daidzein $>$ vanillic acid $>$ ferulic acid, indicating that puerarin was the most abundant in YYTN. The result of this experiment was consistent with that of previous research of our laboratory.

As shown in Table 7, DPPH radical scavenging percentage and FRAP values can reflect the antioxidant activity [44]. The results showed that YYTN had a good antioxidant activity. The literature showed that the antioxidant activity increased with the decrease of $\mathrm{IC}_{50}$ of DPPH radical scavenging percentage and the increase of FRAP values $[45,46]$. According to the PPMCC shown in Table 8, P4, P6, P7, P8, $P 9, P 10, P 12, P 16, P 21, P 23$, and $P 24$ are positively correlated with DPPH scavenging percentage and FRAP values. $P 6$, $P 10$, and $P 12$ were negatively correlated with $\mathrm{IC}_{50}$ of DPPH radical scavenging percentage, while these peaks were positively correlated with FRAP. The order of correlation between peaks and $\mathrm{IC}_{50}$ of $\mathrm{DPPH}$ radical scavenging percentage followed $P 12>P 10>P 6$, and FRAP values followed $P 6>P 12>P 10$. MLRA could eliminate the problem of multicollinearity that may exist among multiple independent variables. Therefore, MLRA analysis showed fewer peaks related to antioxidant activity than PPMCC. By MLRA, P5, P6, P9, P13, P15, P16, P21, and P24 were related to $\mathrm{IC}_{50}$ of DPPH radical scavenging percentage and FRAP values. Among them, P5, P6, P13, P15, and P24 were negatively correlated with $\mathrm{IC}_{50}$ of $\mathrm{DPPH}$ radical scavenging percentage, and $P 5, P 6, P 13$, and $P 24$ were positively correlated with FRAP, which meant that $P 5, P 6, P 13, P 15$, and $P 24$ may have significant antioxidant activity. $P 5$ and $P 13$ were identified by HPLC as vanillic acid and ferulic acid, but $P 6, P 15$, and P24 were not identified in this study. Therefore, 


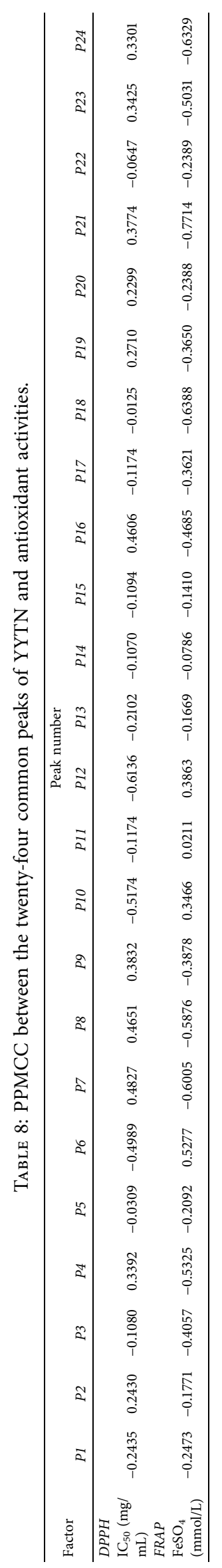


we will further identify these three unknown components and prove whether these five peaks have a greater contribution to the antioxidant activity.

Because of the complexity of the components of traditional Chinese medicines, the chemical fingerprint cannot accurately evaluate the related information between the components and their efficacy $[47,48]$. In this study, the fingerprints of YYTN were established, and no related reports were found in the literature. It was concluded that this study laid a foundation for the establishment of the fingerprint of YYTN. Subsequently, the antioxidant activity of YYTN was tested, which showed that YYTN had a certain extent of antioxidant activity and provided a certain therapeutic idea for the treatment of cardiovascular and cerebrovascular diseases because YYTN has been shown to be effective for cerebral ischemia and cardiac ischemia in animal experiments $[49,50]$. Finally, the components of YYTN related to antioxidant activity were determined by correlation analysis, which may be significant to the research of the therapeutic material basis of YYTN.

Mathematical modeling is used in spectrum-effect relationship of traditional Chinese medicines [34]. For example, the two methods (PPMCC and MLRA) we used in this work indicated that both could establish the spectral correlation model to determine the chromatographic peaks which are closely related to the antioxidant activity. The two antioxidant methods mentioned in this work were also used in a lot of research [51-53]. The results showed that the methods were reliable for the determination of antioxidant activity in vitro. Of course, we will verify this result further in both cell and animal experiments in vivo. Our study not only established the spectrum-effect relationship, but also quantitative analysis of the active components in YYTN compared to other traditional Chinese medicine spectrumeffect correlations [20,34].

In general, the main advantage of this research is that the relationship between HPLC fingerprints and the antioxidant activity of YYTN was established. Main antioxidant components of YYTN are further determined and would contribute to the quality standards of YYTN and to the study of the mechanism of other antioxidant components.

\section{Conclusion}

In the present study, we established the spectrum-effect relationship between the HPLC fingerprints and scavenging activity for DPPH and the total antioxidant activity for FRAP of YYTN. The HPLC analysis was used to build fingerprints of YYTN which contained up to twenty-four common peaks, and the similarity values of these fingerprints were evaluated by similarity analysis. The results showed that the similarity values of nine samples were more than 0.907 , and puerarin was the most abundant chemical constituent in YYTN. The results of the spectrum-effect relationship indicate that peaks $5,6,13,15$, and 24 may be the main components responsible for the antioxidant activity of YYTN. This study shows that the fingerprinting method has been validated; it could provide a reliable and practical method for the consistency of quality of traditional Chinese medicine and other herbal preparations.

\section{Data Availability}

The data used to support the findings of this study are available from the corresponding author upon request.

\section{Conflicts of Interest}

The authors declare no conflicts of interest.

\section{Authors' Contributions}

Yangyang Zhang and Li Yu contributed equally to this work. Yangyang Zhang performed the experiments. Li Yu designed the experiments and drafted the manuscript. Xixi Zhao analyzed the data. Haofang Wan and Yu He helped to revise the manuscript. Haitong Wan and Jiehong Yang conceived the study and revised the manuscript.

\section{Acknowledgments}

This research was funded by the National Natural Science Foundation of China (Grant nos. 81904083, 81973560, and 81630105), key projects of the Natural Science Foundation of Zhejiang Province (Grant no. LZ18H270001), and the Foundation of Zhejiang Chinese Medical University (Grant no. 2018ZJ11).

\section{References}

[1] L.-P. Luo, F.-Q. Cheng, L. Ji, and Y. He-Yong, "Zhongguo Zhong yao za zhi = Zhongguo zhongyao zazhi," China Journal of Chinese Material Medical, vol. 32, pp. 2531-2534, 2007.

[2] H. Jin, F. Hongjin, Y. Jiehong, L. Min, and Z. Yuyan, "Pharmacokinetics of the main ingredient puerarin after intragastric administration of yangyin tongnao granule in rats with cerebral ischemia reperfusion," Chinese Medicine Emergency, vol. 25, no. 6, pp. 945-948, 2016.

[3] Y. Wang, J. Yang, H. Du, H. Zhang, H. Wan, and Y. He, "Yangyin Tongnao granules enhance neurogenesis in the periinfarct area and upregulate brain-derived neurotrophic factor and vascular endothelial growth factor after focal cerebral ischemic infarction in rats," Molecular Biology Reports, vol. 46, no. 4, pp. 3817-3826, 2019.

[4] W. Hong, S. WangShu, and L.-P. Wan, "Zhongguo Zhong xi yi jie he za zhi Zhongguo Zhongxiyi jiehe zazhi," Chinese Journal of Integrated Traditional and Western Medicine, vol. 35, pp. 281-286, 2015.

[5] Y. Jiehong, Z. Yuyan, W. Huafeng, and W. Huafeng, "Effect of yangyin yiqi huoxue recipe on anticoagulant and fibrinolytic function of cultured human umbilical vein endothelial cells," Chinese Journal of Integrated Traditional Chinese and Western Medicine Emergency, no. 1, pp. 3-5, 2008.

[6] R. Yan, H. Yu, C. Lan et al., "Study on the protective mechanism of combination of effective components of yangyin tongnao granule on cerebral ischemia-reperfusion injury in rats," Chinese Journal of Pharmacy, vol. 53, no. 3, pp. 199-204, 2018. 
[7] W. Jiayang, Z. Yangyang, H. Yu et al., "Study on the orthogonal compatibility of the main effective parts of yangyin tongnao granules based on the correlation of pharmacokinetics and pharmacodynamics," Chinese Herbal Medicine, pp. 1-14, 2019.

[8] O. Firuzi, A. Lacanna, R. Petrucci, G. Marrosu, and L. Saso, "Evaluation of the antioxidant activity of flavonoids by "ferric reducing antioxidant power:" assay and cyclic voltammetry," Biochimica et Biophysica Acta (BBA)-General Subjects, vol. 1721, no. 1-3, pp. 174-184, 2005.

[9] A. Salahi, "Screening and evaluation of antioxidant activity of some 1,2,4-triazolo[1,5-a]quinazoline derivatives," Future Medicinal Chemistry, vol. 10, pp. 379-390, 2018.

[10] L. Devanand, "Luthria and sudarsan mukhopadhyay, influence of sample preparation on assay of phenolic acids from eggplant," Journal of Agricultural and Food Chemistry, vol. 54, pp. 1151-1157, 2006.

[11] C.-Y. Lee, "Microfluidic mixing: a review," International Journal of Molecular Sciences, vol. 12, pp. 3941-3949, 2011.

[12] Y. Shang, "Applications of nanotechnology in plant growth and crop protection: a review," Molecules, vol. 24, 2019.

[13] X. Zhao, X. Tianqi, X. Wei et al., "Lyciyunin, a new dimer of feruloyltyramine and five bioactive tyramines from the root of lycium yunnanense kuang," Natural Product Research, vol. 35, no. 3, pp. 447-454, 2021.

[14] W. Yang, "Editorial Board," Journal of Pharmaceutical and Biomedical Analysis, vol. 156, pp. 278-283, 2018.

[15] C. Wu, X. Wang, H. Wang et al., "Extraction optimization, isolation, preliminary structural characterization and antioxidant activities of the cell wall polysaccharides in the petioles and pedicels of Chinese herbal medicine Qian (Euryale ferox Salisb.)," International Journal of Biological Macromolecules, vol. 64, pp. 458-467, 2014.

[16] K. Inoue and H. Yuasa, "Molecular basis for pharmacokinetics and pharmacodynamics of methotrexate in rheumatoid arthritis therapy," Drug Metabolism and Pharmacokinetics, vol. 29, pp. 112-119, 2014.

[17] Q. Li, "Differential and interactive effects of substrate topography and chemistry on human mesenchymal stem cell gene expression," International Journal of Molecular Sciences, vol. 19, 2018.

[18] A. Marchese, R. Barbieri, E. Coppo et al., "Antimicrobial activity of eugenol and essential oils containing eugenol: a mechanistic viewpoint," Critical Reviews in Microbiology, vol. 43, no. 6, pp. 668-689, 2017.

[19] J. Zhang, T. Chen, K. Li, H. Xu, and R. Liang, "Screening active ingredients of rosemary based on spectrum-effect relationships between UPLC fingerprint and vasorelaxant activity using three chemometrics," Journal of Chromatography $B$, vol. 1134-1135, Article ID 121854, 2019.

[20] X. Zhou, "Spectrum-effect relationship between uplc fingerprints and antilung cancer effect of si jun zi tang," EvidenceBased Complementary and Alternative Medicine, vol. 2019, Article ID 7282681, 9 pages, 2019.

[21] L. Xiao, W. Xiaoli, Z. Tingting et al., "Study on spectrum-effect correlation for screening the effective components in fangji huangqi tang basing on ultra-high performance liquid chromatography-mass spectrometry," Phytomedicine: international journal of phytotherapy and phytopharmacology, vol. 47, pp. 81-92, 2018.

[22] M. K.-F. Bader and S. Leuzinger, "Hydraulic coupling of a leafless kauri tree remnant to conspecific hosts," Iscience, vol. 19, pp. 1238-1247, 2019.
[23] N. Han, S. Qiao, G. Yuan, P. Huang, D. Liu, and K. Yue, “A novel Chinese herbal medicine clustering algorithm via artificial bee colony optimization," Artificial Intelligence in Medicine, vol. 101, Article ID 101760, 2019.

[24] K. JP, "Panoramic Radiology: Endodontic Considerations," Panoramic Radiology, vol. 265, pp. 133-142, 2012.

[25] T. E. Bozkurt, "Endocannabinoid system in the airways," Molecules, vol. 24, no. 24, p. 4626, 2019.

[26] M. Diksic, Y. Tohyama, and A. Takada, "Brain net unidirectional uptake of $\alpha$-[14C]methyl-L-tryptophan ( $\alpha$-MTrp) and its correlation with regional serotonin synthesis, tryptophan incorporation into proteins, and permeability surface area products of tryptophan and $\alpha$-MTrp," Neurochemical Research, vol. 25, pp. 1537-1546, 2000.

[27] W. Jinxu, T. Xin, L. Peibo et al., "Bioactive components on immuno-enhancement effects in the traditional chinese medicine shenqi fuzheng injection based on relevance analysis between chemical hplc fingerprints and in vivo biological effects," Journal of Ethnopharmacology, vol. 155, no. 1, pp. 405-415, 2014.

[28] D. Qian, Z. Yaxing, G. Yang, and L. Huang, "Review of chemical constituents in the Genus Lycium (Solanaceae)," Molecules, vol. 22, 2016.

[29] Y Xiong, L Chen, Y Hu, and X Cui, "Uncovering active constituents responsible for different activities of raw and steamed panax notoginseng roots," Frontiers in Pharmacology, vol. 8, p. 745, 2017.

[30] H. Zhang, J. Wang, Y. Chen et al., "Establishing the chromatographic fingerprint of traditional Chinese medicine standard decoction based on quality by design approach: a case study of Licorice," Journal of Separation Science, vol. 42, no. 6, pp. 1144-1154, 2019.

[31] J. Zhou, L. Zhang, Q. Li et al., "Simultaneous optimization for ultrasound-assisted extraction and antioxidant activity of flavonoids from sophora flavescens using response surface methodology," Molecules, vol. 24, no. 1, p. 112, 2018.

[32] N. Echegaray, P. Munekata, J. Centeno et al., "Total phenol content and antioxidant activity of different celta pig carcass locations as affected by the finishing diet," Antioxidants, vol. 10, 2020.

[33] Y. Liu, Q. Meng, R. Chen, J. Wang, S. Jiang, and Y. Hu, “A new method to evaluate the similarity of chromatographic fingerprints: weighted pearson product-moment correlation coefficient," Journal of Chromatographic Science, vol. 42, pp. 545-550, 2004.

[34] M. Liu, Y. Wu, S. Huang, H. Liu, and J. Feng, "Spectrum-effect relationship between HPLC fingerprints and hypolipidemic effect of Curcuma aromatica," Biomedical Chromatography, vol. 32, no. 7, p. e4220, 2018.

[35] L. Xu, W. Zhang, L. Zeng, and J.-O. Jin, "Rehmannia glutinosa polysaccharide induced an anti-cancer effect by activating natural killer cells," International Journal of Biological Macromolecules, vol. 105, pp. 680-685, 2017.

[36] L. Xu, M. Kwak, W. Zhang, L. Zeng, P. C. Lee, and J. O. Jin, "Rehmannia glutinosa polysaccharide induces toll-like receptor 4 dependent spleen dendritic cell maturation and anti-cancer immunity," Oncoimmunology, vol. 6, Article ID e1325981, 2017.

[37] Z. Lei, "Astragaloside IV inhibits the invasion and metastasis of SiHa cervical cancer cells via the TGF- $\beta 1$-mediated PI3K and MAPK pathways," Oncology Reports, vol. 41, pp. 29752986, 2019.

[38] Z. Chunhong, Y. Xiao, W. Jingran et al., "Ethnopharmacology, phytochemistry, pharmacology, toxicology 
and clinical applications of radix astragali," Chinese Journal of Integrative Medicine, vol. 27, no. 3, pp. 229-240, 2019.

[39] C. F. Ng, C. M. Koon, D. W. S. Cheung et al., "The antihypertensive effect of Danshen (Salvia miltiorrhiza) and Gegen (Pueraria lobata) formula in rats and its underlying mechanisms of vasorelaxation," Journal of Ethnopharmacology, vol. 137, no. 3, pp. 1366-1372, 2011.

[40] R. Chen, P. Wu, Z. Cai et al., "Puerariae lobatae radix with chuanxiong rhizoma for treatment of cerebral ischemic stroke by remodeling gut microbiota to regulate the brain-gut barriers," Journal of Nutritional Biochemistry, vol. 65, pp. 101-114, 2019.

[41] L Yun, J Chng, and L. Julia, "Application of Chinese medicine in acute and critical medical conditions," The American Journal of Chinese Medicine, vol. 47, pp. 1419-1444, 2019.

[42] W.-J. Yang, Y.-R. Li, H. Gao et al., "Protective effect of the ethanol extract from Ligusticum chuanxiong rhizome against streptozotocin-induced diabetic nephropathy in mice," Journal of Ethnopharmacology, vol. 227, pp. 166-175, 2018.

[43] H. Jie, J. Xuejing, F. Xiaobin et al., "Ultrasonic extraction, antioxidant and anticancer activities of novel polysaccharides from chuanxiong rhizome," International Journal of Biological Macromolecules, vol. 85, pp. 277-284, 2016.

[44] N. Chaves, A. Santiago, and J. C. Alías, "Quantification of the antioxidant activity of plant extracts: analysis of sensitivity and hierarchization based on the method used," Antioxidants, vol. 9, no. 1, p. 76, 2020.

[45] B. K. Ghimire, E. S. Seong, C. O. Lee et al., "Improved antioxidant activity in transgenic Perilla frutescens plants via overexpression of the $\gamma$-tocopherol methyltransferase $(\gamma$-tmt) gene," Protoplasma, vol. 252, no. 5, pp. 1285-1290, 2015.

[46] B. Santosh, "Parit, nutritional quality and antioxidant activity of wheatgrass (Triticum aestivum) unwrap by proteome profiling and DPPH and FRAP assays," Journal of Food Science, vol. 83, pp. 2127-2139, 2018.

[47] J. Wang, H. Kong, and Z. Yuan, "A novel strategy to evaluate the quality of traditional Chinese medicine based on the correlation analysis of chemical fingerprint and biological effect," Journal of Pharmaceutical and Biomedical Analysis, vol. 89, pp. 130-141, 2014.

[48] S. Wei, C Zhang, and D Zhao, "Discovery of hepatotoxic equivalent combinatorial markers from dioscorea bulbifera tuber by fingerprint-toxicity relationship modeling," Scientific Reports, vol. 8, p. 462, 2018.

[49] W. Yu, Y. Jiehong, H. Yu, Z. Huifen, and W. Haitong, "Protective effect of yangyin tongnao granule on cerebral ischemia/reperfusion injury in rats and its mechanism of inhibiting nf- $\mathrm{kb}$ signaling pathway," Chinese Journal of Traditional Chinese Medicine, vol. 32, no. 8, pp. 3713-3717, 2017.

[50] Z. Xiaoling, C. Shaoyang, X. Lize et al., "Experimental study on the effects of yangyin tongnao granules on dogs' blood pressure and heart rate," Heart Journal, no. 2, pp. 115-120, 2003.

[51] A. Pintatum, S. Laphookhieo, E. Logie, W. V. Berghe, and W. Maneerat, "Chemical composition of essential oils from different parts of Zingiber kerrii Craib and their antibacterial, antioxidant, and tyrosinase inhibitory activities," Biomolecules, vol. 10, no. 2, 2020.

[52] B. Wang, L. Liu, Q. Huang, and Y. Luo, "Quantitative assessment of phenolic acids, flavonoids and antioxidant activities of sixteen jujube cultivars from China," Plant Foods for Human Nutrition, vol. 75, no. 2, pp. 154-160, 2020.

[53] Y. Chen, G. Pan, W. Xu et al., "Spectrum-effect relationship study between HPLC fingerprints and antioxidant activity of Sabia parviflora," Journal of Chromatography B, vol. 1140, Article ID 121970, 2020. 